\title{
Energy- and Regularity-Dependent Stability Estimates for Near-Field Inverse Scattering in Multidimensions
}

\author{
M. I. Isaev \\ Centre de Mathématiques Appliquées, Ecole Polytechnique, 91128 Palaiseau, France \\ Correspondence should be addressed to M. I. Isaev; isaev.m.i@gmail.com
}

Received 20 November 2012; Revised 17 December 2012; Accepted 17 December 2012

Academic Editor: Zindoga Mukandavire

Copyright (C) 2013 M. I. Isaev. This is an open access article distributed under the Creative Commons Attribution License, which permits unrestricted use, distribution, and reproduction in any medium, provided the original work is properly cited.

We prove new global Hölder-logarithmic stability estimates for the near-field inverse scattering problem in dimension $d \geq 3$. Our estimates are given in uniform norm for coefficient difference and related stability efficiently increases with increasing energy and/or coefficient regularity. In addition, a global logarithmic stability estimate for this inverse problem in dimension $d=2$ is also given.

\section{Introduction}

We consider the Schrödinger equation:

$$
L \psi=E \psi, \quad L=-\Delta+v(x), \quad x \in \mathbb{R}^{d}, d \geq 2,
$$

where

$$
\begin{gathered}
v \text { is real-valued, } \quad v \in \mathbb{L}^{\infty}\left(\mathbb{R}^{d}\right), \\
v(x)=O\left(|x|^{-d-\varepsilon}\right), \quad|x| \longrightarrow \infty \text {, for some } \varepsilon>0 .
\end{gathered}
$$

We consider the resolvent $R(E)$ of the Schrödinger operator $L$ in $\mathbb{L}^{2}\left(\mathbb{R}^{d}\right)$ :

$$
R(E)=(L-E)^{-1}, \quad E \in \mathbb{C} \backslash \sigma(L),
$$

where $\sigma(L)$ is the spectrum of $L$ in $\mathbb{L}^{2}\left(\mathbb{R}^{d}\right)$. We assume that $R(x, y, E)$ denotes the Schwartz kernel of $R(E)$ as of an integral operator. We consider also

$$
R^{+}(x, y, E)=R(x, y, E+i 0), \quad x, y \in \mathbb{R}^{d}, E \in \mathbb{R}_{+} .
$$

We recall that in the framework of (1) the function $R^{+}(x, y, E)$ describes scattering of the spherical waves,

$$
R_{0}^{+}(x, y, E)=-\frac{i}{4}\left(\frac{\sqrt{E}}{2 \pi|x-y|}\right)^{(d-2) / 2} H_{(d-2) / 2}^{(1)}(\sqrt{E}|x-y|),
$$

generated by a source at $y$ (where $H_{\mu}^{(1)}$ is the Hankel function of the first kind of order $\mu)$. We recall also that $R^{+}(x, y, E)$ is the Green function for $L-E, E \in \mathbb{R}_{+}$, with the Sommerfeld radiation condition at infinity.

In addition, the function

$$
\begin{aligned}
S^{+}(x, y, E)= & R^{+}(x, y, E)-R_{0}^{+}(x, y, E), \\
& x, y \in \partial B_{r}, \quad E \in \mathbb{R}_{+}, \quad r \in \mathbb{R}_{+}
\end{aligned}
$$

is considered as near-field scattering data for (1), where $B_{r}$ is the open ball of radius $r$ centered at 0 .

We consider, in particular, the following near-field inverse scattering problem for (1).

Problem 1. Given $S^{+}$on $\partial B_{r} \times \partial B_{r}$ for some fixed $r, E \in \mathbb{R}_{+}$, find $v$ on $B_{r}$.

This problem can be considered under the assumption that $v$ is a priori known on $\mathbb{R}^{d} \backslash B_{r}$. Actually, in the present paper we consider Problem 1 under the assumption that $v \equiv 0$ on $\mathbb{R}^{d} \backslash B_{r}$ for some fixed $r \in \mathbb{R}_{+}$. Below in this paper we always assume that this additional condition is fulfilled.

It is well known that the near-field scattering data of Problem 1 uniquely and efficiently determine the scattering amplitude $f$ for (1) at fixed energy $E$, see [1]. Therefore, approaches of [2-12] can be applied to Problem 1 via this reduction. 
In addition, it is also known that the near-field data of Problem 1 uniquely determine the Dirichlet-to-Neumann map in the case when $E$ is not a Dirichlet eigenvalue for operator $L$ in $B_{r}$, see $[8,13]$. Therefore, approaches of $[3,8$, 14-24] can be also applied to Problem 1 via this reduction.

However, in some case it is much more optimal to deal with Problem 1 directly, see, for example, logarithmic stability results of [25] for Problem 1 in dimension $d=3$. A principal improvement of estimates of [25] was given recently in [26]: stability of [26] efficiently increases with increasing regularity of $v$.

Problem 1 can be also considered as an example of illposed problem: see $[27,28]$ for an introduction to this theory.

In the present paper we continue studies of $[25,26]$. We give new global Hölder-logarithmic stability estimates for Problem 1 in dimension $d \geq 3$, see Theorem 1 . Our estimates are given in uniform norm for coefficient difference and related stability efficiently increases with increasing energy and/or coefficient regularity. Results of such a type for the Gel'fand inverse problem were obtained recently in [15] for $d \geq 3$ and in [29] for $d=2$.

The main feature of our new estimates is the explicit dependence on the energy $E$. These estimates consist of two parts, the first is Hölder and the second is logarithmic; when $E$ increases, the logarithmic part decreases and the Hölder part becomes dominant.

In addition, we give also global logarithmic stability estimates for Problem 1 in dimension $d=2$, see Theorem 2 .

\section{Stability Estimates}

We recall that if $v$ satisfies (2) and $\operatorname{supp} v \subset B_{r_{1}}$ for some $r_{1}>0$, then

$$
S^{+}(E) \text { is bounded in } \mathbb{L}^{2}\left(\partial B_{r} \times \partial B_{r}\right) \text { for any } r>r_{1} \text {, }
$$

where $S^{+}(E)$ is the near-field scattering data of $v$ for (1) with $E>0$, for more details see, for example, Section 2 of [25].

2.1. Estimates for $d \geq 3$. In this subsection we assume for simplicity that

$$
v \in \mathbb{W}^{m, 1}\left(\mathbb{R}^{d}\right) \text { for some } m>d, v \text { is real-valued, }
$$$$
\operatorname{supp} v \subset B_{r_{1}} \quad \text { for some } r_{1}>0 \text {, }
$$

where

$$
\mathbb{W}^{m, 1}\left(\mathbb{R}^{d}\right)=\left\{v: \partial^{J} v \in \mathbb{L}^{1}\left(\mathbb{R}^{d}\right),|J| \leq m\right\}, \quad m \in \mathbb{N} \cup 0,
$$

where

$$
\begin{gathered}
J \in(\mathbb{N} \cup 0)^{d}, \quad|J|=\sum_{i=1}^{d} J_{i}, \\
\partial^{J} v(x)=\frac{\partial^{|J|} v(x)}{\partial x_{1}^{J_{1}} \cdots \partial x_{d}^{J_{d}}} .
\end{gathered}
$$

Let

$$
\|v\|_{m, 1}=\max _{|J| \leq m}\left\|\partial^{J} v\right\|_{\mathbb{L}^{1}\left(\mathbb{R}^{d}\right)}
$$

Note that $(8) \Rightarrow(2)$.

Theorem 1. Let $E>0$ and $r>r_{1}$ be given constants. Let dimensiond $\geq 3$ and potentials $v_{1}$, $v_{2}$ satisfy (8). Let $\left\|v_{j}\right\|_{m, 1} \leq$ $N, j=1,2$, for some $N>0$. Let $S_{1}^{+}(E)$ and $S_{2}^{+}(E)$ denote the near-field scattering data for $v_{1}$ and $v_{2}$, respectively. Then for $\tau \in(0,1)$ and any $s \in\left[0, s^{*}\right]$ the following estimate holds:

$$
\begin{aligned}
\| v_{2}- & v_{1} \|_{L^{\infty}\left(B_{r}\right)} \\
& \leq C_{1}(1+E)^{5 / 2} \delta^{\tau}+C_{2}(1+E)^{\left(s-s^{*}\right) / 2}\left(\ln \left(3+\delta^{-1}\right)\right)^{-s},
\end{aligned}
$$

where $s^{*}=(m-d) / d, \delta=\left\|S_{1}^{+}(E)-S_{2}^{+}(E)\right\|_{\mathbb{L}^{2}\left(\partial B_{r} \times \partial B_{r}\right)}$, and constants $C_{1}, C_{2}>0$ depend only on $N, m, d, r, \tau$.

Proof of Theorem 1 is given in Section 5. This proof is based on results presented in Sections 3 and 4.

2.2. Estimates for $d=2$. In this subsection we assume for simplicity that

$$
\begin{aligned}
& v \text { is real-valued, } \quad v \in C^{2}\left(\bar{B}_{r_{1}}\right) \text {, } \\
& \operatorname{supp} v \subset B_{r_{1}} \text { for some } r_{1}>0 .
\end{aligned}
$$

Note also that $(13) \Rightarrow(2)$.

Theorem 2. Let $E>0$ and $r>r_{1}$ be given constants. Let dimension $d=2$ and potentials $v_{1}, v_{2}$ satisfy (13). Let $\left\|v_{j}\right\|_{C^{2}\left(B_{r}\right)} \leq N, \quad j=1,2$, for some $N>0$. Let $S_{1}^{+}(E)$ and $S_{2}^{+}(E)$ denote the near-field scattering data for $v_{1}$ and $v_{2}$, respectively. Then

$$
\left\|v_{1}-v_{2}\right\|_{L^{\infty}\left(B_{r}\right)} \leq C_{3}\left(\ln \left(3+\delta^{-1}\right)\right)^{-3 / 4}\left(\ln \left(3 \ln \left(3+\delta^{-1}\right)\right)\right)^{2},
$$

where $\delta=\left\|S_{1}^{+}(E)-S_{2}^{+}(E)\right\|_{\mathbb{L}^{2}\left(\partial B_{r} \times \partial B_{r}\right)}$ and constant $C_{3}>0$ depends only on $N, m, r$.

Proof of Theorem 2 is given in Section 7. This proof is based on results presented in Sections 3 and 6.

\subsection{Concluding Remarks}

Remark 3. The logarithmic stability estimates for Problem 1.1 of $[25,26]$ follow from estimate (12) for $d=3$ and $s=s^{*}$. Apparently, using the methods of $[19,20]$ it is possible to improve estimate (12) for $s^{*}=m-d$.

Remark 4. In the same way as in $[25,26]$ for dimesnsion $d=$ 3 , using estimates (12) and (14), one can obtain logarithmic stability estimates for the reconstruction of a potential $v$ from the inverse scattering amplitude $f$ for any $d \geq 2$. 
Remark 5. Actually, in the proof of Theorem 1 we obtain the following estimate (see formula (57)):

$$
\begin{aligned}
\left\|v_{1}-v_{2}\right\|_{\mathbb{L}^{\infty}\left(B_{r}\right)} \leq & C_{4}(1+E)^{2} \sqrt{E+\rho^{2}} e^{2 \rho(r+1)} \delta \\
& +C_{5}\left(E+\rho^{2}\right)^{-(m-d) / 2 d}
\end{aligned}
$$

where constants $C_{4}, C_{5}>0$ depend only on $N, m, d, r$ and the parameter $\rho>0$ is such that $E+\rho^{2}$ is sufficiently large: $E+\rho^{2} \geq C_{6}(N, r, m)$. Estimate of Theorem 1 follows from estimate (15).

\section{Alessandrini-Type Identity for Near-Field Scattering}

In this section we always assume that assumptions of Theorems 1 and 2 are fulfilled (in the cases of dimension $d \geq 3$ and $d=2$, resp.).

Consider the operators $\widehat{R}_{j}, j=1,2$, defined as follows

$$
\left(\widehat{R}_{j} \phi\right)(x)=\int_{\partial B_{r}} R_{j}^{+}(x, y, E) \phi(y) d y, \quad x \in \partial B_{r}, \quad j=1,2 .
$$

Note that

$$
\left\|\widehat{R}_{1}-\widehat{R}_{2}\right\|_{\mathbb{L}^{2}\left(\partial B_{r}\right)} \leq\left\|S_{1}^{+}(E)-S_{2}^{+}(E)\right\|_{\mathbb{L}^{2}\left(\partial B_{r}\right) \times \mathbb{L}^{2}\left(\partial B_{r}\right)} .
$$

We recall that (see [25]) for any functions $\phi_{1}, \phi_{2} \in C\left(\mathbb{R}^{d}\right)$, sufficiently regular in $\mathbb{R}^{d} \backslash \partial B_{r}$ and satisfying

$$
\begin{gathered}
-\Delta \phi+v(x) \phi=E \phi, \quad \text { in } \mathbb{R}^{d} \backslash \partial B_{r} \\
\lim _{|x| \rightarrow+\infty}|x|^{(d-1) / 2}\left(\frac{\partial}{\partial|x|} \phi-i \sqrt{E} \phi\right)=0
\end{gathered}
$$

with $v=v_{1}$ and $v=v_{2}$, respectively, the following identity holds:

$$
\begin{aligned}
& \int_{B_{r}}\left(v_{2}-v_{1}\right) \phi_{1} \phi_{2} d x \\
& \quad=\int_{\partial B_{r}}\left(\frac{\partial \phi_{1}}{\partial v_{+}}-\frac{\partial \phi_{1}}{\partial \nu_{-}}\right)\left[\left(\widehat{R}_{1}-\widehat{R}_{2}\right)\left(\frac{\partial \phi_{2}}{\partial v_{+}}-\frac{\partial \phi_{2}}{\partial \nu_{-}}\right)\right] d x,
\end{aligned}
$$

where where $v_{+}$and $v_{-}$are the outward and inward normals to $\partial B_{r}$, respectively.

Remark 6. The identity (19) is similar to the Alessandrini identity (see Lemma 1 of [14]), where the Dirichlet-toNeumann maps are considered instead of operators $\widehat{R}_{j}$.

To apply identity (19) to our considerations, we use also the following lemma.
Lemma 7. Let $E, r>0$ and $d \geq 2$. Then, there is a positive constant $C_{7}$ (depending only on $r$ and $d$ ) such that for any $\phi \in$ $C\left(\mathbb{R}^{d} \backslash B_{r}\right)$ satisfying

$$
\begin{gathered}
-\Delta \phi=E \phi, \quad \text { in } \mathbb{R}^{d} \backslash \bar{B}_{r} \\
\lim _{|x| \rightarrow+\infty}|x|^{(d-1) / 2}\left(\frac{\partial}{\partial|x|} \phi-i \sqrt{E} \phi\right)=0, \\
\left.\phi\right|_{\partial B_{r}} \in \mathbb{H}^{1}\left(\partial B_{r}\right),
\end{gathered}
$$

the following inequality holds:

$$
\left\|\left.\frac{\partial \phi}{\partial \nu_{+}}\right|_{\partial B_{r}}\right\|_{\mathbb{L}^{2}\left(\partial B_{r}\right)} \leq C_{7}(1+E)\left\|\left.\phi\right|_{\partial B_{r}}\right\|_{\mathbb{H}^{1}\left(\partial B_{r}\right)^{\prime}},
$$

where $\mathbb{H}^{1}\left(\partial B_{r}\right)$ denotes the standard Sobolev space on $\partial B_{r}$.

The proof of Lemma 7 is given in Section 8 .

\section{Faddeev Functions}

In dimension $d \geq 3$, we consider the Faddeev functions $h, \psi$, $G($ see $[6,8,30,31])$ :

$$
h(k, l)=(2 \pi)^{-d} \int_{\mathbb{R}^{d}} e^{-i l x} v(x) \psi(x, k) d x,
$$

where $k, l \in \mathbb{C}^{d}, k^{2}=l^{2}, \operatorname{Im} k=\operatorname{Im} l \neq 0$,

$$
\begin{gathered}
\psi(x, k)=e^{i k x}+\int_{\mathbb{R}^{d}} G(x-y, k) v(y) \psi(y, k) d y, \\
G(x, k)=e^{i k x} g(x, k), \\
g(x, k)=-(2 \pi)^{-d} \int_{\mathbb{R}^{d}} \frac{e^{i \xi x} d \xi}{\xi^{2}+2 k \xi},
\end{gathered}
$$

where $x \in \mathbb{R}^{d}, k \in \mathbb{C}^{d}, \operatorname{Im} k \neq 0, d \geq 3$,

One can consider (22), (23) assuming that

$$
\begin{gathered}
v \text { is a sufficiently regular function on } \mathbb{R}^{d} \\
\text { with suffucient decay at infinity. }
\end{gathered}
$$

For example, in connection with Theorem 1, we consider (22), (23) assuming that

$$
v \in \mathbb{L}^{\infty}\left(B_{r}\right), \quad v \equiv 0 \text { on } \mathbb{R} \backslash B_{r} .
$$

We recall that (see $[6,8,30,31])$

$$
\left(\Delta+k^{2}\right) G(x, k)=\delta(x), \quad x \in \mathbb{R}^{d}, k \in \mathbb{C}^{d} \backslash \mathbb{R}^{d},
$$

formula (23) at fixed $k$ is considered as an equation for

$$
\psi=e^{i k x} \mu(x, k),
$$

where $\mu$ is sought in $\mathbb{L}^{\infty}\left(\mathbb{R}^{d}\right)$; as a corollary of (23), (24), and (27), $\psi$ satisfies (1) for $E=k^{2} ; h$ of (22) is a generalized "scattering" amplitude. 
In addition, $h, \psi, G$ in their zero energy restriction, that is for $E=0$ were considered for the first time in [32]. The Faddeev functions $h, \psi, G$ were, actually, rediscovered in [32].

Let

$$
\begin{gathered}
\Sigma_{E}=\left\{k \in \mathbb{C}^{d}: k^{2}=k_{1}^{2}+\cdots+k_{d}^{2}=E\right\}, \\
\Theta_{E}=\left\{k \in \Sigma_{E}, l \in \Sigma_{E}: \operatorname{Im} k=\operatorname{Im} l\right\}, \\
|k|=\left(|\operatorname{Re} k|^{2}+|\operatorname{Im} k|^{2}\right)^{1 / 2} .
\end{gathered}
$$

Let

$$
\begin{gathered}
v \text { satisfy }(8), \quad\|v\|_{m, 1} \leq N, \\
\widehat{v}(p)=(2 \pi)^{-d} \int_{\mathbb{R}^{d}} e^{i p x} v(x) d x, \quad p \in \mathbb{R}^{d},
\end{gathered}
$$

then we have that

$$
\mu(x, k) \longrightarrow 1 \text { as }|k| \longrightarrow \infty
$$

and, for any $\sigma>1$,

$$
|\mu(x, k)|+|\nabla \mu(x, k)| \leq \sigma \quad \text { for }|k| \geq \lambda_{1}(N, m, d, r, \sigma),
$$

where $x \in \mathbb{R}^{d}, k \in \Sigma_{E}$;

$$
\begin{gathered}
\widehat{v}(p)=\lim _{\substack{(k, l) \in \Theta_{E}, k-l=p \\
|\operatorname{Im} k|=|\operatorname{Im} l| \rightarrow \infty}} h(k, l), \quad \text { for any } p \in \mathbb{R}^{d}, \\
|\widehat{v}(p)-h(k, l)| \\
\leq \frac{c_{1}(m, d, r) N^{2}}{\left(E+\rho^{2}\right)^{1 / 2}}, \quad \text { for }(k, l) \in \Theta_{E}, p=k-l, \\
|\operatorname{Im} k|=|\operatorname{Im} l|=\rho, \quad E+\rho^{2} \geq \lambda_{2}(N, m, d, r), \\
p^{2} \leq 4\left(E+\rho^{2}\right) .
\end{gathered}
$$

Results of the type (31), (32) go back to [32]. For more information concerning (32) see estimate (4.11) of [33]. Results of the type (33), (34) (with less precise right-hand side in (34)) go back to [6]. Estimate (34) follows, for example, from formulas (23), (22), and the estimate

$$
\begin{aligned}
& \left\|\Lambda^{-s} g(k) \Lambda^{-s}\right\|_{\mathbb{L}^{2}\left(\mathbb{R}^{d}\right) \rightarrow \mathbb{L}^{2}\left(\mathbb{R}^{d}\right)} \\
& \quad=O\left(|k|^{-1}\right) \quad \text { as }|k| \longrightarrow \infty, k \in \mathbb{C}^{d} \backslash \mathbb{R}^{d},
\end{aligned}
$$

for $s>1 / 2$, where $g(k)$ denotes the integral operator with the Schwartz kernel $g(x-y, k)$ and $\Lambda$ denotes the multiplication operator by the function $\left(1+|x|^{2}\right)^{1 / 2}$. Estimate (35) was formulated, first, in [34] for $d \geq 3$. Concerning proof of (35), see [35].
In addition, we have that

$$
\begin{aligned}
& h_{2}(k, l)-h_{1}(k, l) \\
& =(2 \pi)^{-d} \int_{\mathbb{R}^{d}} \psi_{1}(x,-l)\left(v_{2}(x)-v_{1}(x)\right) \psi_{2}(x, k) d x \\
& \text { for }(k, l) \in \Theta_{E}, \quad|\operatorname{Im} k|=|\operatorname{Im} l| \neq 0, \\
& \text { and } v_{1}, v_{2} \text { satisfying }(25),
\end{aligned}
$$

and, under assumptions of Theorem 1 ,

$$
\begin{array}{r}
\left|\widehat{v}_{1}(p)-\widehat{v}_{2}(p)-h_{1}(k, l)+h_{2}(k, l)\right| \\
\leq \frac{c_{2}(m, d, r) N\left\|v_{1}-v_{2}\right\|_{\mathbb{L}^{\infty}\left(B_{r}\right)}}{\left(E+\rho^{2}\right)^{1 / 2}}
\end{array}
$$

for $(k, l) \in \Theta_{E}, \quad p=k-l, \quad|\operatorname{Im} k|=|\operatorname{Im} l|=\rho$,

$$
E+\rho^{2} \geq \lambda_{3}(N, m, d, r), \quad p^{2} \leq 4\left(E+\rho^{2}\right),
$$

where $h_{j}, \psi_{j}$ denote $h$ and $\psi$ of (22) and (23) for $v=v_{j}, j=$ 1,2 .

Formula (36) was given in [36]. Estimate (37) was given for example in [15].

\section{Proof of Theorem 1}

Let

$$
\begin{aligned}
& \mathbb{L}_{\mu}^{\infty}\left(\mathbb{R}^{d}\right)=\left\{u \in \mathbb{L}^{\infty}\left(\mathbb{R}^{d}\right):\|u\|_{\mu}<+\infty\right\}, \\
& \|u\|_{\mu}=\operatorname{ess} \sup _{p \in \mathbb{R}^{d}}(1+|p|)^{\mu}|u(p)|, \quad \mu>0 .
\end{aligned}
$$

Note that

$$
\begin{gathered}
w \in \mathbb{W}^{m, 1}\left(\mathbb{R}^{d}\right) \Longrightarrow \widehat{w} \in \mathbb{L}_{\mu}^{\infty}\left(\mathbb{R}^{d}\right) \cap C\left(\mathbb{R}^{d}\right), \\
\|\widehat{w}\|_{\mu} \leq c_{3}(m, d)\|w\|_{m, 1}, \quad \text { for } \mu=m,
\end{gathered}
$$

where $\mathbb{W}^{m, 1}, \mathbb{L}_{\mu}^{\infty}$ are the spaces of (9), (38),

$$
\widehat{w}(p)=(2 \pi)^{-d} \int_{\mathbb{R}^{d}} e^{i p x} w(x) d x, \quad p \in \mathbb{R}^{d} .
$$

Using the inverse Fourier transform formula

$$
w(x)=\int_{\mathbb{R}^{d}} e^{-i p x} \widehat{w}(p) d p, \quad x \in \mathbb{R}^{d},
$$

we have that

$$
\begin{aligned}
\left\|v_{1}-v_{2}\right\|_{\mathbb{L}^{\infty}\left(B_{r}\right)} & \leq \sup _{x \in \bar{B}_{r}}\left|\int_{\mathbb{R}^{d}} e^{-i p x}\left(\widehat{v}_{2}(p)-\widehat{v}_{1}(p)\right) d p\right| \\
& \leq I_{1}(\kappa)+I_{2}(\kappa), \quad \text { for any } \kappa>0,
\end{aligned}
$$

where

$$
\begin{aligned}
& I_{1}(\kappa)=\int_{|p| \leq \kappa}\left|\widehat{v}_{2}(p)-\widehat{v}_{1}(p)\right| d p, \\
& I_{2}(\kappa)=\int_{|p| \geq \kappa}\left|\widehat{v}_{2}(p)-\widehat{v}_{1}(p)\right| d p .
\end{aligned}
$$


Using (39), we obtain that

$$
\left|\widehat{v}_{2}(p)-\widehat{v}_{1}(p)\right| \leq 2 c_{3}(m, d) N(1+|p|)^{-m}, \quad p \in \mathbb{R}^{d}
$$

Let

$$
c_{4}=\int_{p \in \mathbb{R}^{d},|p|=1} d p
$$

Combining (43), (44), we find that, for any $\kappa>0$,

$$
I_{2}(\kappa) \leq 2 c_{3}(m, d) N c_{4} \int_{\kappa}^{+\infty} \frac{d t}{t^{m-d+1}} \leq \frac{2 c_{3}(m, d) N c_{4}}{m-d} \frac{1}{\kappa^{m-d}}
$$

Due to (37), we have that

$$
\begin{aligned}
\left|\widehat{v}_{2}(p)-\widehat{v}_{1}(p)\right| \leq & \left|h_{2}(k, l)-h_{1}(k, l)\right| \\
& +\frac{c_{2}(m, d, r) N\left\|v_{1}-v_{2}\right\|_{\mathbb{L}^{\infty}\left(B_{r}\right)}}{\left(E+\rho^{2}\right)^{1 / 2}},
\end{aligned}
$$

for $(k, l) \in \Theta_{E}, \quad p=k-l, \quad|\operatorname{Im} k|=|\operatorname{Im} l|=\rho$,

$$
E+\rho^{2} \geq \lambda_{3}(N, m, d, r), \quad p^{2} \leq 4\left(E+\rho^{2}\right) .
$$

Let

$$
\delta=\left\|S_{1}^{+}(E)-S_{2}^{+}(E)\right\|_{\mathbb{L}^{2}\left(\partial B_{r} \times \partial B_{r}\right)}
$$

Combining (17), (19), and (36), we get that

$$
\begin{array}{r}
\left|h_{2}(k, l)-h_{1}(k, l)\right| \\
\leq \delta\left\|\frac{\partial \phi_{1}}{\partial v_{+}}-\frac{\partial \phi_{1}}{\partial v_{-}}\right\|_{\mathbb{L}^{2}\left(B_{r}\right)}\left\|\frac{\partial \phi_{2}}{\partial v_{+}}-\frac{\partial \phi_{2}}{\partial v_{-}}\right\|_{\mathbb{L}^{2}\left(B_{r}\right)}, \\
(k, l) \in \Theta_{E}, \quad|\operatorname{Im} k|=|\operatorname{Im} l| \neq 0,
\end{array}
$$

where $\phi_{j}, j=1,2$, denotes the solution of (18) with $v=v_{j}$, satisfying

$$
\phi_{j}(x)=\psi_{j}(x, k), \quad \text { for } x \in \bar{B}_{r}
$$

Using (21), (32), and the fact that $C^{1}\left(\partial B_{r}\right) \subset \mathbb{H}^{1}\left(\partial B_{r}\right)$, we find that

$$
\begin{aligned}
& \left\|\frac{\partial \phi_{j}}{\partial \nu_{+}}-\frac{\partial \phi_{j}}{\partial \nu_{-}}\right\|_{\mathbb{L}^{2}\left(B_{r}\right)} \\
& \quad \leq \sigma c_{5}(r, d)(1+E) \exp (|\operatorname{Im} k|(r+1)), \\
& k \in \Sigma_{E}, \quad|k| \geq \lambda_{1}(N, m, d, r, \sigma), \quad j=1,2 .
\end{aligned}
$$

Here and bellow in this section the constant $\sigma$ is the same that in (32).
Combining (49) and (51), we obtain that

$$
\begin{gathered}
\left|h_{2}(k, l)-h_{1}(k, l)\right| \leq c_{5}^{2} \sigma^{2}(1+E)^{2} e^{2 \rho(r+1)} \delta, \\
\text { for }(k, l) \in \Theta_{E}, \quad \rho=|\operatorname{Im} k|=|\operatorname{Im} l|, \\
E+\rho^{2} \geq \lambda_{1}^{2}(N, m, d, r, \sigma) .
\end{gathered}
$$

Using (47), (52), we get that

$$
\begin{gathered}
\left|\widehat{v}_{2}(p)-\widehat{v}_{1}(p)\right| \leq c_{5}^{2} \sigma^{2}(1+E)^{2} e^{2 \rho(r+1)} \delta \\
+\frac{c_{2}(m, d, r) N\left\|v_{1}-v_{2}\right\|_{\mathbb{L}^{\infty}\left(B_{1}\right)}}{\left(E+\rho^{2}\right)^{1 / 2}} \\
p \in \mathbb{R}^{d}, \quad p^{2} \leq 4\left(E+\rho^{2}\right), \quad E+\rho^{2} \geq \max \left\{\lambda_{1}^{2}, \lambda_{3}\right\} .
\end{gathered}
$$

Let

$$
\varepsilon=\left(\frac{1}{2 c_{2}(m, d, r) N c_{6}}\right)^{1 / d}, \quad c_{6}=\int_{p \in \mathbb{R}^{d},|p| \leq 1} d p,
$$

and $\lambda_{4}(N, m, d, r, \sigma)>0$ be such that

$$
E+\rho^{2} \geq \lambda_{4}(N, m, d, r, \sigma)
$$

$$
\Longrightarrow\left\{\begin{array}{l}
E+\rho^{2} \geq \lambda_{1}^{2}(N, m, d, r, \sigma), \\
E+\rho^{2} \geq \lambda_{3}(N, m, d, r), \\
\left(\varepsilon\left(E+\rho^{2}\right)^{1 / 2 d}\right)^{2} \leq 4\left(E+\rho^{2}\right) .
\end{array}\right.
$$

Using (43), (53), we get that

$$
\begin{gathered}
I_{1}(\kappa) \leq c_{6} \kappa^{d}\left(c_{5}^{2} \sigma^{2}(1+E)^{2} e^{2 \rho(r+1)} \delta\right. \\
+\frac{\left.c_{2}(m, d, r) N\left\|v_{1}-v_{2}\right\|_{\mathbb{L}^{\infty}\left(B_{1}\right)}\right)}{\left.\left(E+\rho^{2}\right)^{1 / 2}\right)} \\
\kappa>0, \kappa^{2} \leq 4\left(E+\rho^{2}\right), \\
E+\rho^{2} \geq \lambda_{4}(N, m, d, r, \sigma) .
\end{gathered}
$$

Combining (42), (46), and (56) for $\kappa=\varepsilon\left(E+\rho^{2}\right)^{1 / 2 d}$ and (55), we get that

$$
\begin{aligned}
&\left\|v_{1}-v_{2}\right\|_{\mathbb{L}^{\infty}\left(B_{r}\right)} \\
& \leq c_{7}(N, m, d, r, \sigma)(1+E)^{2} \sqrt{E+\rho^{2}} e^{2 \rho(r+1)} \delta \\
&+ c_{8}(N, m, d)\left(E+\rho^{2}\right)^{-(m-d) / 2 d} \\
&+ \frac{1}{2}\left\|v_{1}-v_{2}\right\|_{\mathbb{L}^{\infty}\left(B_{r}\right)}, \\
& E+\rho^{2} \geq \lambda_{4}(N, m, d, r, \sigma) .
\end{aligned}
$$

Let $\tau^{\prime} \in(0,1)$,

$$
\beta=\frac{1-\tau^{\prime}}{2(r+1)}, \quad \rho=\beta \ln \left(3+\delta^{-1}\right),
$$


and $\delta_{1}=\delta_{1}\left(N, m, d, \sigma, r, \tau^{\prime}\right)>0$ be such that

$$
\delta \in\left(0, \delta_{1}\right) \Longrightarrow\left\{\begin{array}{c}
E+\left(\beta \ln \left(3+\delta^{-1}\right)\right)^{2} \\
\geq \lambda_{4}(N, m, d, r, \sigma) \\
E+\left(\beta \ln \left(3+\delta^{-1}\right)\right)^{2} \\
\leq(1+E)\left(\beta \ln \left(3+\delta^{-1}\right)\right)^{2}
\end{array}\right.
$$

Then for the case when $\delta \in\left(0, \delta_{1}\right)$, due to (57), we have that

$$
\begin{aligned}
\frac{1}{2} \| v_{1} & -v_{2} \|_{\mathbb{L}^{\infty}\left(B_{r}\right)} \\
\leq & c_{7}(1+E)^{2}\left(E+\left(\beta \ln \left(3+\delta^{-1}\right)\right)^{2}\right)^{1 / 2}\left(3+\delta^{-1}\right)^{2 \beta(r+1)} \delta \\
& +c_{8}\left(E+\left(\beta \ln \left(3+\delta^{-1}\right)\right)^{2}\right)^{-(m-d) / 2 d} \\
= & c_{7}(1+E)^{2}\left(E+\left(\beta \ln \left(3+\delta^{-1}\right)\right)^{2}\right)^{1 / 2}(1+3 \delta)^{1-\tau^{\prime}} \delta^{\tau^{\prime}} \\
& +c_{8}\left(E+\left(\beta \ln \left(3+\delta^{-1}\right)\right)^{2}\right)^{-(m-d) / 2 d} .
\end{aligned}
$$

Combining (59) and (60), we obtain that for $s \in\left[0, s^{*}\right], \tau \in$ $\left(0, \tau^{\prime}\right)$ and $\delta \in\left(0, \delta_{1}\right)$ the following estimate holds:

$$
\begin{aligned}
\left\|v_{2}-v_{1}\right\|_{L^{\infty}\left(B_{r}\right)} \leq & c_{9}(1+E)^{5 / 2} \delta^{\tau} \\
& +c_{10}(1+E)^{\left(s-s^{*}\right) / 2}\left(\ln \left(3+\delta^{-1}\right)\right)^{-s},
\end{aligned}
$$

where $s^{*}=(m-d) / d$ and $c_{9}, c_{10}>0$ depend only on $N, m$, $d, r, \sigma, \tau^{\prime}$, and $\tau$.

Estimate (61) in the general case (with modified $c_{9}$ and $c_{10}$ ) follows from (61) for $\delta \leq \delta_{1}\left(N, m, d, \sigma, r, \tau^{\prime}\right)$ and the property that

$$
\left\|v_{j}\right\|_{\mathbb{L}^{\infty}\left(B_{r}\right)} \leq c_{11}(m, d) N
$$

This completes the proof of (12).

\section{Buckhgeim-Type Analogs of the Faddeev Functions}

Let us identify $\mathbb{R}^{2}$ with $\mathbb{C}$ and use coordinates $z=x_{1}+i x_{2}$, $\bar{z}=x_{1}-i x_{2}$, where $\left(x_{1}, x_{2}\right) \in \mathbb{R}^{2}$. Following [21-23, 37], we consider the functions $G_{z_{0}}, \psi_{z_{0}}, \widetilde{\psi}_{z_{0}}, \delta h_{z_{0}}$ going back to Buckhgeim's paper [3] and being analogs of the Faddeev functions:

$$
\begin{aligned}
\psi_{z_{0}}(z, \lambda)= & e^{\lambda\left(z-z_{0}\right)^{2}} \\
& +\int_{B_{r}} G_{z_{0}}(z, \zeta, \lambda) v(\zeta) \psi_{z_{0}}(\zeta, \lambda) d \operatorname{Re} \zeta d \operatorname{Im} \zeta, \\
\widetilde{\psi}_{z_{0}}(z, \lambda)= & e^{\bar{\lambda}\left(\bar{z}-\bar{z}_{0}\right)^{2}} \\
& +\int_{B_{r}} \overline{G_{z_{0}}(z, \zeta, \lambda)} v(\zeta) \widetilde{\psi}_{z_{0}}(\zeta, \lambda) d \operatorname{Re} \zeta d \operatorname{Im} \zeta,
\end{aligned}
$$

$$
\begin{aligned}
G_{z_{0}}(z, \zeta, \lambda)=\frac{1}{4 \pi^{2}} \int_{B_{r}} & \frac{e^{-\lambda\left(\eta-z_{0}\right)^{2}+\bar{\lambda}\left(\bar{\eta}-\bar{z}_{0}\right)^{2}} d \operatorname{Re} \eta d \operatorname{Im} \eta}{(z-\eta)(\bar{\eta}-\bar{\zeta})} \\
& \times e^{\lambda\left(z-z_{0}\right)^{2}-\bar{\lambda}\left(\bar{\zeta}-\bar{z}_{0}\right)^{2}}, \\
& z=x_{1}+i x_{2}, \quad z_{0} \in B_{r}, \quad \lambda \in \mathbb{C},
\end{aligned}
$$

where $v$ satisfies (13),

$$
\begin{aligned}
\delta h_{z_{0}}(\lambda)= & \int_{B_{r}} \widetilde{\psi}_{z_{0}, 1}(z,-\lambda)\left(v_{2}(z)-v_{1}(z)\right) \\
& \times \psi_{z_{0}, 2}(z, \lambda) d \operatorname{Re} z d \operatorname{Im} z, \quad \lambda \in \mathbb{C},
\end{aligned}
$$

where $v_{1}, v_{2}$ satisfy (13) and $\widetilde{\psi}_{z_{0}, 1}, \psi_{z_{0}, 2}$ denote $\widetilde{\psi}_{z_{0}}, \psi_{z_{0}}$ of (63) for $v=v_{1}$ and $v=v_{2}$, respectively.

We recall that (see $[21,22])$ :

(i) the function $G_{z_{0}}$ satisfies the equations

$$
\begin{aligned}
& 4 \frac{\partial^{2}}{\partial z \partial \bar{z}} G_{z_{0}}(z, \zeta, \lambda)=\delta(z-\zeta), \\
& 4 \frac{\partial^{2}}{\partial \zeta \partial \bar{\zeta}} G_{z_{0}}(z, \zeta, \lambda)=\delta(z-\zeta),
\end{aligned}
$$

where $z, z_{0}, \zeta \in B_{r}, \lambda \in \mathbb{C}$, and $\delta$ is the Dirac delta function;

(ii) formulas (63) at fixed $z_{0}$ and $\lambda$ are considered as equations for $\psi_{z_{0}}, \widetilde{\psi}_{z_{0}}$ in $L^{\infty}\left(B_{r}\right)$;

(iii) as a corollary of (63), (64), (66), the functions $\psi_{z_{0}}, \widetilde{\psi}_{z_{0}}$ satisfy (1) in $B_{r}$ for $E=0$ and $d=2$;

(iv) the function $\delta h_{z_{0}}$ is similar to the right side of (36).

Let potentials $v, v_{1}, v_{2} \in C^{2}\left(\bar{B}_{r}\right)$, and

$$
\begin{gathered}
\|v\|_{C^{2}\left(\bar{B}_{r}\right)} \leq N, \quad\left\|v_{j}\right\|_{C^{2}\left(\bar{B}_{r}\right)} \leq N, \quad j=1,2, \\
\left.\left(v_{1}-v_{2}\right)\right|_{\partial B_{r}}=0,\left.\quad \frac{\partial}{\partial \nu}\left(v_{1}-v_{2}\right)\right|_{\partial B_{r}}=0
\end{gathered}
$$


then we have that

$$
\begin{gathered}
\psi_{z_{0}}(z, \lambda)=e^{\lambda\left(z-z_{0}\right)^{2}} \mu_{z_{0}}(z, \lambda), \\
\widetilde{\psi}_{z_{0}}(z, \lambda)=e^{\bar{\lambda}\left(\bar{z}-\bar{z}_{0}\right)^{2}} \widetilde{\mu}_{z_{0}}(z, \lambda), \\
\mu_{z_{0}}(z, \lambda) \longrightarrow 1, \quad \tilde{\mu}_{z_{0}}(z, \lambda) \longrightarrow 1 \quad \text { as }|\lambda| \longrightarrow \infty
\end{gathered}
$$

and, for any $\sigma>1$,

$$
\begin{gathered}
\left|\mu_{z_{0}}(z, \lambda)\right|+\left|\nabla \mu_{z_{0}}(z, \lambda)\right| \leq \sigma, \\
\left|\tilde{\mu}_{z_{0}}(z, \lambda)\right|+\left|\nabla \tilde{\mu}_{z_{0}}(z, \lambda)\right| \leq \sigma,
\end{gathered}
$$

where $\nabla=\left(\partial / \partial x_{1}, \partial / \partial x_{2}\right), z=x_{1}+i x_{2}, z_{0} \in B_{r}, \lambda \in \mathbb{C}$, $|\lambda| \geq \rho_{1}(N, r, \sigma)$;

$$
\begin{aligned}
& v_{2}\left(z_{0}\right)-v_{1}\left(z_{0}\right) \\
& =\lim _{\lambda \rightarrow \infty} \frac{2}{\pi}|\lambda| \delta h_{z_{0}}(\lambda) \quad \text { for any } z_{0} \in B_{r} \\
& \left|v_{2}\left(z_{0}\right)-v_{1}\left(z_{0}\right)-\frac{2}{\pi}\right| \lambda\left|\delta h_{z_{0}}(\lambda)\right| \\
& \leq \frac{c_{12}(N, r)(\ln (3|\lambda|))^{2}}{|\lambda|^{3 / 4}} \\
& \quad \text { for } z_{0} \in B_{r}, \quad|\lambda| \geq \rho_{2}(N, r) .
\end{aligned}
$$

Formulas (68) can be considered as definitions of $\mu_{z_{0}}, \widetilde{\mu}_{z_{0}}$. Formulas (69), (71) were given in [21, 22] and go back to [3]. Estimates (70a) and (70b) were proved in [33]. Estimate (72) was obtained in [21, 37].

\section{Proof of Theorem 2}

We suppose that $\widetilde{\psi}_{z_{0}, 1}(\cdot,-\lambda), \psi_{z_{0}, 2}(\cdot, \lambda), \delta h_{z_{0}}(\lambda)$ are defined as in Section 6 but with $v_{j}-E$ in place of $v_{j}, j=1,2$. Note that functions $\widetilde{\psi}_{z_{0}, 1}(\cdot,-\lambda), \psi_{z_{0}, 2}(\cdot, \lambda)$ satisfy (1) in $B_{r}$ with $v=v_{j}$, $j=1,2$, respectively. We also use the notation $N_{E}=N+E$. Then, using (72), we have that

$$
\begin{gathered}
\left|v_{2}\left(z_{0}\right)-v_{1}\left(z_{0}\right)-\frac{2}{\pi}\right| \lambda\left|\delta h_{z_{0}}(\lambda)\right| \\
\leq \frac{c_{12}\left(N_{E}, r\right)(\ln (3|\lambda|))^{2}}{|\lambda|^{3 / 4}},
\end{gathered}
$$$$
\text { for } z_{0} \in B_{r},|\lambda| \geq \rho_{2}\left(N_{E}, r\right) \text {. }
$$

Let

$$
\delta=\left\|S_{1}^{+}(E)-S_{2}^{+}(E)\right\|_{\mathbb{L}^{2}\left(\partial B_{r} \times \partial B_{r}\right)} .
$$

Combining (17), (19), and (65), we get that

$$
\begin{array}{r}
\left|\delta h_{z_{0}}(\lambda)\right| \leq \delta\left\|\frac{\partial \phi_{1}}{\partial v_{+}}-\frac{\partial \phi_{1}}{\partial v_{-}}\right\|_{\mathbb{L}^{2}\left(B_{r}\right)}\left\|\frac{\partial \phi_{2}}{\partial \nu_{+}}-\frac{\partial \phi_{2}}{\partial v_{-}}\right\|_{\mathbb{L}^{2}\left(B_{r}\right)}, \\
(k, l) \in \Theta_{E}, \quad|\operatorname{Im} k|=|\operatorname{Im} l| \neq 0,
\end{array}
$$

where $\phi_{j}, j=1,2$, denotes the solution of (18) with $v=v_{j}$, satisfying

$$
\begin{aligned}
& \phi_{1}(x)=\widetilde{\psi}_{z_{0}, 1}(x,-\lambda), \\
& \phi_{2}(x)=\psi_{z_{0}, 2}(x, \lambda), \quad \text { for } x \in \bar{B}_{r} .
\end{aligned}
$$

Using (21), (70a), and (70b) and the fact that $C^{1}\left(\partial B_{r}\right) \subset$ $\mathbb{H}^{1}\left(\partial B_{r}\right)$, we find that

$$
\begin{aligned}
\left\|\frac{\partial \phi_{j}}{\partial \nu_{+}}-\frac{\partial \phi_{j}}{\partial \nu_{-}}\right\|_{\mathbb{L}^{2}\left(B_{r}\right)} \leq \sigma c_{13}(r)(1+E) \exp \left(|\lambda|\left(4 r^{2}+4 r\right)\right), \\
\lambda \in \mathbb{C}, \quad|\lambda| \geq \rho_{1}\left(N_{E}, r, \sigma\right), \quad j=1,2 .
\end{aligned}
$$

Here and bellow in this section the constant $\sigma$ is the same that in (70a) and (70b).

Combining (75), (77), we obtain that

$$
\begin{array}{r}
\left|\delta h_{z_{0}}(\lambda)\right| \leq c_{14}(E, r, \sigma) \exp \left(|\lambda|\left(8 r^{2}+8 r\right)\right) \delta, \\
\lambda \in \mathbb{C}, \quad|\lambda| \geq \rho_{1}\left(N_{E}, r, \sigma\right) .
\end{array}
$$

Using (73) and (78), we get that

$$
\begin{aligned}
\left|v_{2}\left(z_{0}\right)-v_{1}\left(z_{0}\right)\right| \leq & c_{14}(E, r, \sigma) \exp \left(|\lambda|\left(8 r^{2}+8 r\right)\right) \delta \\
& +\frac{c_{12}\left(N_{E}, r\right)(\ln (3|\lambda|))^{2}}{|\lambda|^{3 / 4}}
\end{aligned}
$$$$
z_{0} \in B_{r}, \quad \lambda \in \mathbb{C}, \quad|\lambda| \geq \rho_{3}\left(N_{E}, r, \sigma\right)=\max \left\{\rho_{1}, \rho_{2}\right\} .
$$

We fix some $\tau \in(0,1)$ and let

$$
\beta=\frac{1-\tau}{8 r^{2}+8 r}, \quad \lambda=\beta \ln \left(3+\delta^{-1}\right),
$$

where $\delta$ is so small that $|\lambda| \geq \rho_{3}\left(N_{E}, r, \sigma\right)$. Then due to (79), we have that

$$
\begin{aligned}
\| v_{1}- & v_{2} \|_{\mathbb{L}^{\infty}\left(B_{r}\right)} \\
\leq & c_{14}(E, r, \sigma)\left(3+\delta^{-1}\right)^{\beta\left(8 r^{2}+8 r\right)} \delta \\
& +c_{12}\left(N_{E}, r\right) \frac{\left(\ln \left(3 \beta \ln \left(3+\delta^{-1}\right)\right)\right)^{2}}{\left(\beta \ln \left(3+\delta^{-1}\right)\right)^{3 / 4}} \\
= & c_{14}(E, r, \sigma)(1+3 \delta)^{1-\tau} \delta^{\tau} \\
& +c_{12}\left(N_{E}, r\right) \beta^{-3 / 4} \frac{\left(\ln \left(3 \beta \ln \left(3+\delta^{-1}\right)\right)\right)^{2}}{\left(\ln \left(3+\delta^{-1}\right)\right)^{3 / 4}},
\end{aligned}
$$

where $\tau, \beta$, and $\delta$ are the same as in (80).

Using (81), we obtain that

$$
\begin{aligned}
\| v_{1}- & v_{2} \|_{\mathbb{L}^{\infty}\left(B_{r}\right)} \\
\leq & c_{15}(N, E, r, \sigma)\left(\ln \left(3+\delta^{-1}\right)\right)^{-3 / 4} \\
& \times\left(\ln \left(3 \ln \left(3+\delta^{-1}\right)\right)\right)^{2},
\end{aligned}
$$


for $\delta=\left\|S_{1}^{+}(E)-S_{2}^{+}(E)\right\|_{\mathbb{L}^{2}\left(\partial B_{r} \times \partial B_{r}\right)} \leq \delta_{2}\left(N_{E}, r, \sigma\right)$, where $\delta_{2}$ is a sufficiently small positive constant. Estimate (82) in the general case (with modified $c_{15}$ ) follows from (82) for $\delta \leq$ $\delta_{2}\left(N_{E}, r, \sigma\right)$ and the property that $\left\|v_{j}\right\|_{\mathbb{L}^{\infty}\left(B_{r}\right)} \leq N$.

This completes the proof of (14).

\section{Proof of Lemma 7}

In this section we assume for simplicity that $r=1$ and therefore $\partial B_{r}=\mathbb{S}^{d-1}$.

We fix an orthonormal basis in $\mathbb{L}^{2}\left(\partial B_{r}\right)$ :

$$
\left\{f_{j p}: j \geq 0 ; 1 \leq p \leq p_{j}\right\},
$$

$f_{j p}$ is a spherical harmonic of degree $j$,

where $p_{j}$ is the dimension of the space of spherical harmonics of order $j$,

$$
p_{j}=\left(\begin{array}{c}
j+d-1 \\
d-1
\end{array}\right)-\left(\begin{array}{c}
j+d-3 \\
d-1
\end{array}\right)
$$

where

$$
\begin{gathered}
\left(\begin{array}{l}
n \\
k
\end{array}\right)=\frac{n(n-1) \cdots(n-k+1)}{k !}, \quad \text { for } n \geq 0, \\
\left(\begin{array}{l}
n \\
k
\end{array}\right)=0, \quad \text { for } n<0 .
\end{gathered}
$$

The precise choice of $f_{j p}$ is irrelevant for our purposes. Besides orthonormality, we only need $f_{j p}$ to be the restriction of a homogeneous harmonic polynomial of degree $j$ to the sphere $\partial B_{r}$ and so $|x|^{j} f_{j p}(x|| x \mid)$ is harmonic pn $\mathbb{R}^{d}$. In the Sobolev spaces $\mathbb{H}^{s}\left(\partial B_{r}\right)$ the norm is defined by

$$
\left\|\sum_{j, p} c_{j p} f_{j p}\right\|_{\mathbb{H}^{s}\left(\partial B_{r}\right)}^{2}=\sum_{j, p}(1+j)^{2 s}\left|c_{j p}\right|^{2} .
$$

The solution $\phi$ of the exterior Dirichlet problem

$$
\begin{gathered}
-\Delta \phi=E \phi, \quad \text { in } \mathbb{R}^{d} \backslash \bar{B}_{r}, \\
\lim _{|x| \rightarrow+\infty}|x|^{(d-1) / 2}\left(\frac{\partial}{\partial|x|} \phi-i \sqrt{E} \phi\right)=0, \\
\left.\phi\right|_{\partial B_{r}}=u \in \mathbb{H}^{1}\left(\partial B_{r}\right),
\end{gathered}
$$

can be expressed in the following form (see, e.g., $[1,38]$ ):

$$
\phi=\sum_{j, p} c_{j p} \phi_{j p}
$$

where $c_{j p}$ are expansion coefficients of $u$ in the basis $\left\{f_{j p}\right.$ : $\left.j \geq 0 ; 1 \leq p \leq p_{j}\right\}$, and

$\phi_{j p}$ denotes the solution of (89) with $u=f_{j p}$,

$$
\begin{gathered}
\phi_{j p}(x)=h_{j p}(|x|) f_{j p}\left(\frac{x}{|x|}\right), \\
h_{j p}(|x|)=|x|^{-(d-2) / 2} \frac{H_{j+((d-2) / 2)}^{(1)}(\sqrt{E}|x|)}{H_{j+((d-2) / 2)}^{(1)}(\sqrt{E})},
\end{gathered}
$$

where $H_{\mu}^{(1)}$ is the Hankel function of the first kind. Let

$$
\phi_{j p}^{0}(x)=|x|^{-j-d+2} f_{j p}\left(\frac{x}{|x|}\right) .
$$

Note that $\phi_{j p}^{0}$ is harmonic in $\mathbb{R}^{d} \backslash\{0\}$ and

$$
\begin{gathered}
\lim _{|x| \rightarrow+\infty}|x|^{(d-1) / 2}\left(\frac{\partial}{\partial|x|} \phi_{j p}^{0}-i \sqrt{E} \phi_{j p}^{0}\right) \\
=0, \quad \text { for } j+\frac{d-3}{2}>0 .
\end{gathered}
$$

Using the Green formula and the radiation condition for $\phi_{j p}, \phi_{j p}^{0}$, we get that

$$
\begin{array}{rl}
\int_{\mathbb{R}^{d} \backslash B_{r}} & E \phi_{j p} \phi_{j p}^{0} d x \\
= & \int_{\mathbb{R}^{d} \backslash B_{r}}\left(\Delta \phi_{j p}^{0} \phi_{j p}-\Delta \phi_{j p} \phi_{j p}^{0}\right) d x \\
= & \int_{\partial B_{r}}\left(\frac{\partial \phi_{j p}^{0}}{\partial \nu_{+}} \phi_{j p}-\frac{\partial \phi_{j p}}{\partial \nu_{+}} \phi_{j p}^{0}\right) d x, \text { for } j+\frac{d-3}{2}>0 .
\end{array}
$$

Due to (89) and (90), we have that

$$
\begin{aligned}
\left|\int_{\partial B_{r}} \frac{\partial \phi_{j p}^{0}}{\partial \nu_{+}} \phi_{j p} d x\right| & =(j+d-2) \int_{\partial B_{r}} f_{j p}^{2} d x \\
& =j+d-2 .
\end{aligned}
$$

Using also the following property of the Hankel function of the first kind (see, e.g., [39]):

$\left|H_{\mu}^{(1)}(x)\right|$ is a decreasing function of $x$, for $x \in \mathbb{R}_{+}, \mu \in \mathbb{R}$, 
we get that

$$
\begin{aligned}
\left|\int_{\mathbb{R}^{d} \backslash B_{r}} \phi_{j p} \phi_{j p}^{0} d x\right| & =\left|\int_{1}^{+\infty} t^{-j-d+2} h_{j p}(t) t^{d-1} d t\right| \\
& =\left|\int_{1}^{+\infty} t^{-j-(d / 2)} \frac{H_{j+((d-2) / 2)}^{(1)}(\sqrt{E} t)}{H_{j+((d-2) / 2)}^{(1)}(\sqrt{E})} d t\right| \\
& \leq \int_{1}^{+\infty} t^{-j-(d / 2)} d t=\frac{1}{j+(d / 2)-1} \\
& \leq 2, \quad \text { for } j+\frac{d-3}{2}>0 .
\end{aligned}
$$

Combining (89), (90), (92), (93), and (95), we obtain that

$$
\begin{array}{r}
\left|\int_{\partial B_{r}} \frac{\partial \phi_{j p}^{0}}{\partial \nu_{+}} \phi_{j p} d x\right|=\left|\frac{h_{j p}^{\prime}(r)}{h_{j p}(r)}\right| \leq j+d-2+2 E \\
\text { for } j+\frac{d-3}{2}>0 .
\end{array}
$$

Let consider the cases when $j+((d-3) / 2) \leq 0$.

Case $1(j=0, d=2)$. Using the property $d H_{0}^{(1)}(t) / d t=$ $-H_{1}^{(1)}(t)$, we get that

$$
\frac{h_{j p}^{\prime}(r)}{h_{j p}(r)}=\sqrt{E} \frac{H_{1}^{(1)}(\sqrt{E})}{H_{0}^{(1)}(\sqrt{E})} .
$$

We recall that functions $H_{0}^{(1)}$ and $H_{1}^{(1)}$ have the following asymptotic forms (see, e.g. [39]):

$$
\begin{array}{cc}
H_{0}^{(1)}(t) \sim \frac{2 i}{\pi} \ln \left(\frac{t}{2}\right), \quad \text { as } t \longrightarrow+0, \\
H_{0}^{(1)}(t) \sim \sqrt{\frac{2}{\pi t}} e^{i(t-\pi / 4)}, \quad \text { as } t \longrightarrow+\infty, \\
H_{1}^{(1)}(t) \sim-\frac{i}{\pi}\left(\frac{2}{t}\right), \quad \text { as } t \longrightarrow+0, \\
H_{1}^{(1)}(t) \sim \sqrt{\frac{2}{\pi t}} e^{i(t-3 \pi / 4)}, \quad \text { as } t \longrightarrow+\infty .
\end{array}
$$

Using (94) and (98), we get that for some $c>0$

$$
\frac{H_{1}^{(1)}(t)}{H_{0}^{(1)}(t)} \leq c\left(1+\frac{1}{t}\right) .
$$

Combining (97) and (99), we obtain that for $j=0, d=2$

$$
\left|\frac{h_{j p}^{\prime}(r)}{h_{j p}(r)}\right| \leq c(1+\sqrt{E}) .
$$

Case $2(j=0, d=3)$. We have that

$$
H_{j+((d-2) / 2)}^{(1)}(t)=\sqrt{\frac{2}{\pi t}} e^{i(t-\pi / 2)} .
$$

Using (89) and (101), we get that for $j=0, d=3$

$$
\frac{h_{j p}^{\prime}(r)}{h_{j p}(r)}=-1+i \sqrt{E} .
$$

Combining (86)-(89), (96), (100), and (102), we get that for some constant $c^{\prime}=c^{\prime}(d)>0$ :

$$
\begin{aligned}
\left\|\left.\frac{\partial \phi}{\partial \nu_{+}}\right|_{\partial B_{r}}\right\|_{\mathbb{L}^{2}\left(\partial B_{r}\right)}^{2} & =\sum_{j, p} c_{j p}^{2}\left|\frac{h_{j p}^{\prime}(r)}{h_{j p}(r)}\right|^{2} \\
& \leq c^{\prime}(1+E)^{2} \sum_{j, p}(1+j)^{2} c_{j p}^{2} .
\end{aligned}
$$

Using (86) and (103), we obtain (21).

\section{Acknowledgment}

This work was partially supported by FCP Kadry no. 14.A18.21.0866.

\section{References}

[1] Ju. M. Berezanskiî, "The uniqueness theorem in the inverse problem of spectral analysis for the Schrödinger equation," Trudy Moskovskogo Matematičeskogo Obščestva, vol. 7, pp. 1-62, 1958 (Russian).

[2] N. V. Alekseenko, V. A. Burov, and O. D. Rumyantseva, "Solution of the three-dimensional acoustic inverse scattering problem. the modified Novikov algorithm," Akusticheskiu Zhurnal, vol. 54, no. 3, pp. 469-482, 2008 (Russian), English translation in Acoustical Physics, vol. 54, no. 3, pp. 407-419, 2008.

[3] A. L. Bukhgeim, "Recovering a potential from Cauchy data in the two-dimensional case," Journal of Inverse and Ill-Posed Problems, vol. 16, no. 1, pp. 19-33, 2008.

[4] V. A. Burov, N. V. Alekseenko, and O. D. Rumyantseva, "Multifrequency generalization of the Novikov algorithm for the two-dimensional inverse scattering problem," Acoustical Physics, vol. 55, no. 6, pp. 843-856, 2009.

[5] G. Eskin and J. Ralston, "Inverse scattering problem for the Schrödinger equation with magnetic potential at a fixed energy," Communications in Mathematical Physics, vol. 173, no. 1, pp. 199-224, 1995.

[6] R. G. Novikov and G. M. Khenkin, "The $\bar{\partial}$-equation in the multidimensional inverse scattering problem," Uspekhi Matematicheskikh Nauk, vol. 42, no. 3(255), pp. 93-152, 1987 (Russian), English translation in Russian Mathematical Surveys, vol. 42, no. 3, pp.109-180, 1987.

[7] M. I. Isaev, "Exponential instability in the inverse scattering problem on the energy interval," to appear in Funktsional'nyi Analiz i ego Prilozheniya, http://arxiv.org/abs/1012.5526.

[8] R. G. Novikov, "A multidimensional inverse spectral problem for the equation $-\Delta \psi+(v(x)-E u(x)) \psi=0$," Funktsional'ny 
Analiz i ego Prilozheniya, vol. 22, no. 4, pp. 11-22, 1988, English translation in Functional Analysis and Its Applications, vol. 22, pp. 263-272, 1988.

[9] R. G. Novikov, "The inverse scattering problem at fixed energy for the three-dimensional Schrödinger equation with an exponentially decreasing potential," Communications in Mathematical Physics, vol. 161, no. 3, pp. 569-595, 1994.

[10] R. G. Novikov, "Approximate solution of the inverse problem of quantum scattering theory with fixed energy in dimension 2," Trudy Matematicheskogo Instituta Imeni V. A. Steklova, vol. 225, pp. 301-318, 1999 (Russian), English translation in Proceedings of the Steklov Institute of Mathematic, no. 2(225), pp. 285-302, 1999.

[11] R. G. Novikov, “The $\bar{\partial}$-approach to approximate inverse scattering at fixed energy in three dimensions," IMRP. International Mathematics Research Papers, no. 6, pp. 287-349, 2005.

[12] P. Stefanov, "Stability of the inverse problem in potential scattering at fixed energy," Annales de l'Institut Fourier, vol. 40, no. 4, pp. 867-884, 1990.

[13] A. I. Nachman, "Reconstructions from boundary measurements," Annals of Mathematics, vol. 128, no. 3, pp. 531-576, 1988.

[14] G. Alessandrini, "Stable determination of conductivity by boundary measurements," Applicable Analysis, vol. 27, no. 1-3, pp. 153-172, 1988.

[15] M. I. Isaev and R. G. Novikov, "Energy and regularity dependent stability estimates for the Gel'fand inverse problem in multidimensions," Journal of Inverse and III-posed Problems, vol. 20, no. 3, pp. 313-325, 2012.

[16] V. Isakov, "Increasing stability for the Schrödinger potential from the Dirichlet-to-Neumann map," Discrete and Continuous Dynamical Systems. Series S, vol. 4, no. 3, pp. 631-640, 2011.

[17] N. Mandache, "Exponential instability in an inverse problem for the Schrödinger equation," Inverse Problems, vol. 17, no. 5, pp. 1435-1444, 2001.

[18] R. G. Novikov, "Formulae and equations for finding scattering data from the Dirichlet-to-Neumann map with nonzero background potential," Inverse Problems, vol. 21, no. 1, pp. 257-270, 2005.

[19] R. G. Novikov, "An effectivization of the global reconstruction in the Gel'fand-Calderón inverse problem in three dimensions," in Imaging Microstructures, vol. 494 of Contemp. Math., pp. 161-184, American Mathematical Society, Providence, RI, USA, 2009.

[20] R. G. Novikov, "New global stability estimates for the Gel'fandCalderon inverse problem," Inverse Problems, vol. 27, no. 1, Article ID 015001, 21 pages, 2011.

[21] R. Novikov and M. Santacesaria, "A global stability estimate for the Gel'fand-Calderón inverse problem in two dimensions," Journal of Inverse and Ill-Posed Problems, vol. 18, no. 7, pp. 765-785, 2010.

[22] R. G. Novikov and M. Santacesaria, "Global uniqueness and reconstruction for the multi-channel Gel'fand-Calderón inverse problem in two dimensions," Bulletin des Sciences Mathématiques, vol. 135, no. 5, pp. 421-434, 2011.

[23] R. Novikov and M. Santacesaria, "Monochromatic reconstruction algorithms for two-dimensional multi-channel inverse problems," International Mathematics Research Notes, 2012.

[24] J. Sylvester and G. Uhlmann, "A global uniqueness theorem for an inverse boundary value problem," Annals of Mathematics, vol. 125, no. 1, pp. 153-169, 1987.
[25] P. Hähner and T. Hohage, "New stability estimates for the inverse acoustic inhomogeneous medium problem and applications," SIAM Journal on Mathematical Analysis, vol. 33, no. 3, pp. 670-685, 2001.

[26] M. I. Isaev and R. G. Novikov, "New global stability estimates for monochromatic inverse acoustic scattering," http://arxiv.org/abs/1210.3821.

[27] L. Beilina and M. V. Klibanov, Approximate Global Convergence and Adaptivity for Coefficient Inverse Problems, Springer, New York, NY, USA, 2012.

[28] M. M. Lavrent'ev, V. G. Romanov, and S. P. Shishatskii, IllPosed Problems of Mathematical Physics and Analysis, vol. 64 of Translations of Mathematical Monographs, American Mathematical Society, Providence, RI, USA, 1986.

[29] M. Santacesaria, "Stability estimates for an inverse problem for the Schrödinger equation at negative energy in two dimensions," Applicable Analysis, 2012.

[30] L. D. Faddeev, "Growing solutions of the Schrödinger equation," Doklady Akademii Nauk SSSR, vol. 165, no. 3, pp. 514-517, 1965 (Russian), English translation in Soviet Physics. Doklady, vol. 10, pp. 1033-1035, 1966.

[31] L. D. Faddeev, "The inverse problem in the quantum theory of scattering. II," Current Problems in Mathematics, vol. 3, pp. 93-180, 259, 1974 (Russian), English translation in Journal of Soviet Mathematics, vol. 5, pp. 334-396, 1976.

[32] R. Beals and R. R. Coifman, "Multidimensional inverse scatterings and nonlinear partial differential equations," in Pseudodifferential Operators and Applications (Notre Dame, Ind., 1984), vol. 43 of Proc. Sympos. Pure Math., pp. 45-70, American Mathematical Society, Providence, RI, USA, 1985.

[33] M. I. Isaev and R. G. Novikov, "Stability estimates for determination of potential from the impedance boundary map," Algebra and Analysis, 2013, http://128.84.158.119/abs/1112.3728v2.

[34] R. B. Lavine and A. I. Nachman, "On the inverse scattering transform for the $n$-dimensional Schrödinger operator," in Topics in Soliton Theory and Exactly Solvable Nonlinear Equations (Oberwolfach, 1986), pp. 33-44, World Scientific, Singapore, 1987.

[35] R. Weder, "Generalized limiting absorption method and multidimensional inverse scattering theory," Mathematical Methods in the Applied Sciences, vol. 14, no. 7, pp. 509-524, 1991.

[36] R. G. Novikov, “'̄-method with nonzero background potential. Application to inverse scattering for the two-dimensional acoustic equation," Communications in Partial Differential Equations, vol. 21, no. 3-4, pp. 597-618, 1996.

[37] M. Santacesaria, "Global stability for the multi-channel Gel'fandCalderon inverse problem in two dimensions," Bulletin des Sciences Mathématiques, vol. 136, no. 7, pp. 731-744, 2012.

[38] D. Colton and R. Kress, Inverse Acoustic and Electromagnetic Scattering Theory, vol. 93 of Applied Mathematical Sciences, Springer, Berlin, Germany, 2nd edition, 1998.

[39] G. N. Watson, A Treatise on the Theory of Bessel Functions, Cambridge University Press, Cambridge, UK, 1944. 


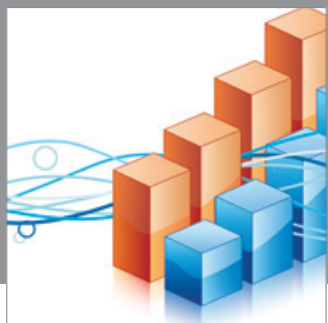

Advances in

Operations Research

mansans

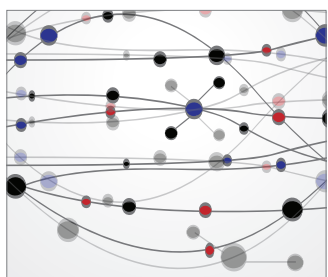

The Scientific World Journal
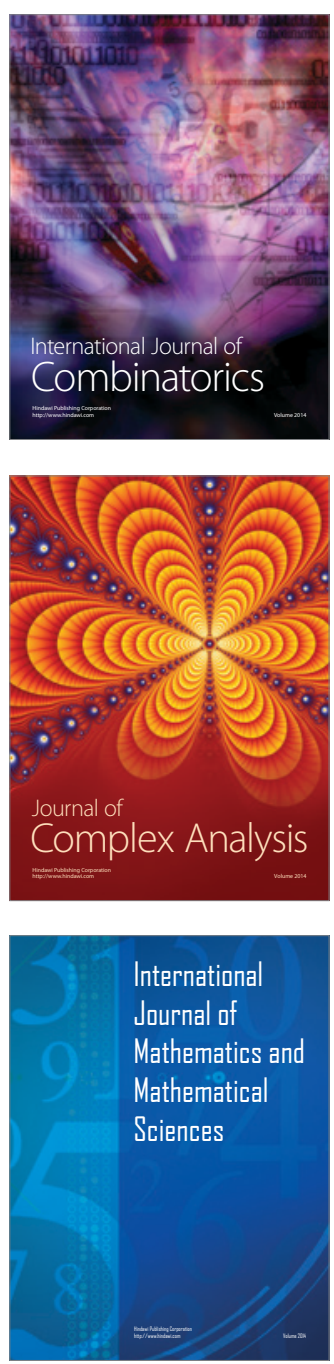
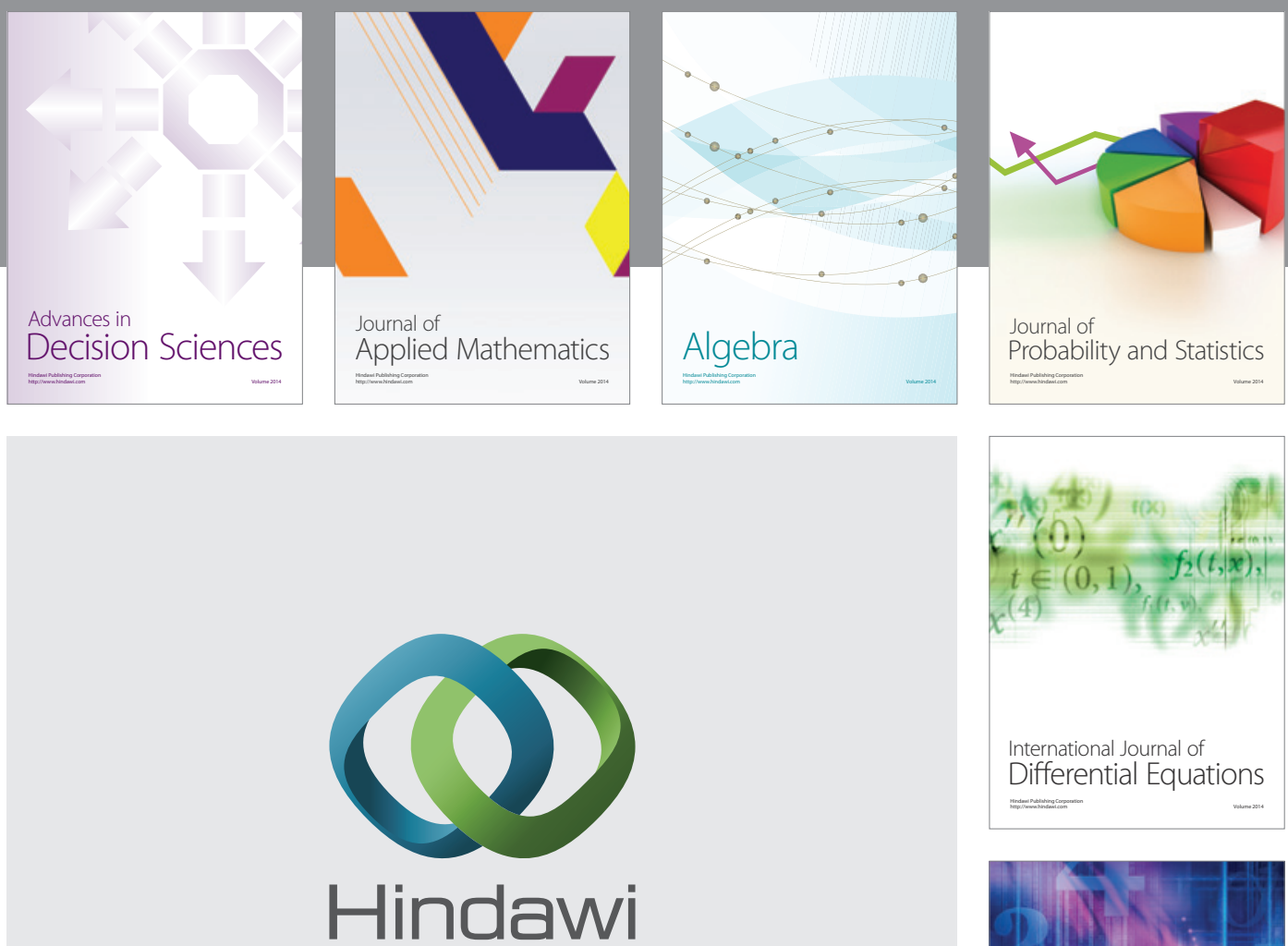

Submit your manuscripts at http://www.hindawi.com
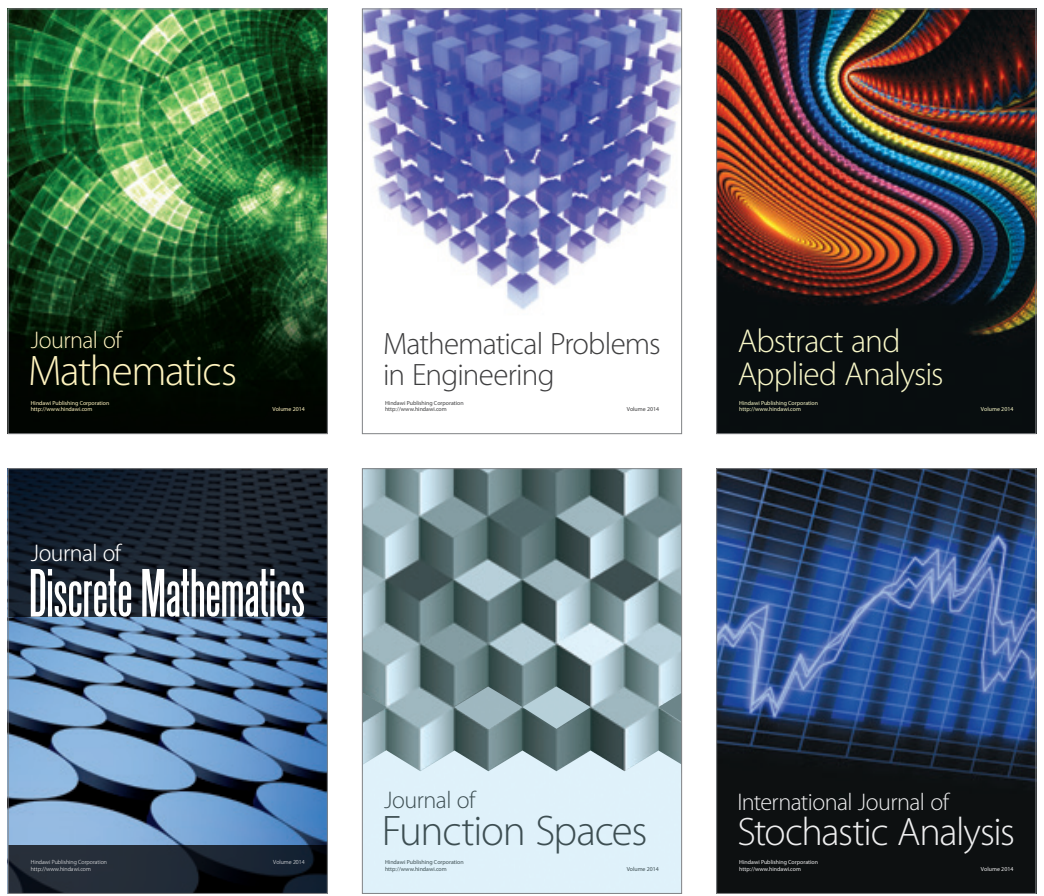

Journal of

Function Spaces

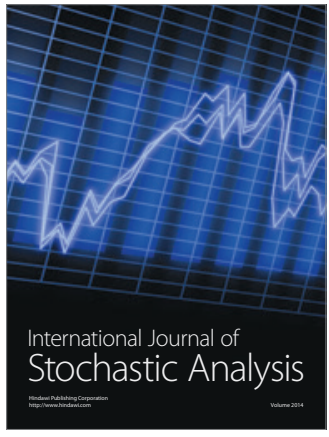

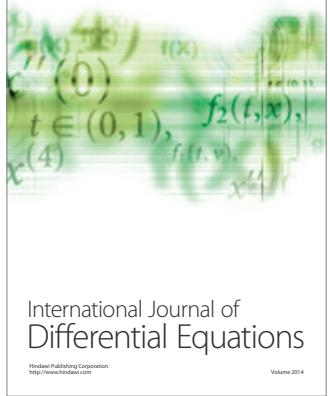
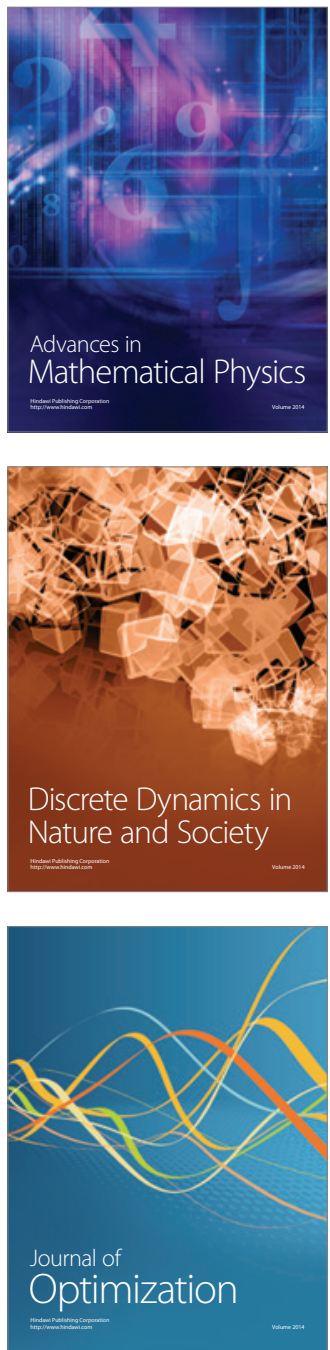